\title{
The effect of stem cells in congenital heart disease
}

\author{
Daina M Chase and Vincent S Gallicchio* \\ Department of Biological Sciences, College of Science, Clemson University, Clemson, SC, USA
}

\begin{abstract}
Congenital Heart Disease (CHD) affects 1 in 100 babies born each year in the United States and is the most prevalent congenital anomaly. Birth prevalence of CHD worldwide varies but the estimate is 8 per 1,000 live births. This is because of the differences in income and access to health care being limited in some parts of the world. Within the first year of life, those with CHD require cardiac intervention quickly. With that in mind, this disease lays a huge burden on the lives of the newborn and their family by putting an enormous emotional and financial strain on them at a vulnerable time. Many CHD patients need open-heart surgery, which is where the chest is cut open and surgery is performed on the heart while the patient is put on cardiopulmonary bypass. Even though this procedure has significantly progressed over the years, it is a heavily invasive surgery that comes with many risks and complications including infection, bleeding, inadequate perfusion of organs or tissues. Since cardiac surgery is the most common reason for admission to pediatric intensive care, there are a large amount of people that experience this burden and these risks. Because of this variability to current treatment options, newer treatments are constantly being developed and tested in clinical trials. However, invasive surgeries remain the first line treatment because of the surgical advancement over the years.

Traditional heart surgery can now be performed with small incisions or minimally invasive heart surgery as an alternative. Therefore, stem cell therapy is beginning to be researched and investigated for the repair of the injured heart in these diseases. Research of stem cell therapy in adults with heart disease has been investigated and has paved way for treating the pediatric population with congenital heart disease. The promising potential of stem cell therapy will provide patients with a different, non-invasive treatment option. The effects of stem cell therapy will promote cardiac repair and recovery. It is the intent of this review to provide an update concerning the existing published evidence of research as well as the need for further investigation of the use of stem cells to treat congenital heart disease.
\end{abstract}

\begin{abstract}
Abbreviations and Symbols: CHD: Congenital Heart Disease; ESCs: Embryonic Stem Cells; hESCs: Human embryonic stem cells; iPSCs: Induced Pluripotent Stem Cells; hiPSCs: Human induced pluripotent stem cells; HLHS: Hypoplastic left heart syndrome; BMSCs: Bone marrow stem cells; hUCBSs: human umbilical cord blood cells; BMNCs: bone marrow mononuclear cells; CSCs: cardiac stem cells; hCSCs: human cardiac stem cells; MSCs: Mesenchymal stem cells; CPCs: Cardiac progenitor cells; ESHF: end- stage heart failure; CDCs: cardiosphere-derived cells; RVEF: right ventricular ejection fraction; AFSC: Amniotic fluid-derived stem cells; pMSCs: placental mesenchymal stem cells; GDF-15: growth differentiation factor 15; RV: right ventricle; LV: left ventricle; ntMSCs: neonatal thymus mesenchymal stem cells; TOF: Tetralogy of Fallot; UCMNC: umbilical cord blood mononuclear cells; UCB: umbilical cord blood; LMSCs: Longerverson mesenchymal stem cells; CM: cardiomyocutes.
\end{abstract}

\section{Introduction}

Stem cells are characterized by having the ability to differentiate into specific cell types. These characteristics include self-renewal and the ability to differentiate into a specialized adult cell type. The two main classes of stem cells include pluripotent and multipotent. Pluripotent are stem cells that can become any cell in the adult body. Multipotent stem cells are more restricted in that they have a more limited ability to differentiate. Stem cells have the ability to build every tissue in the human body which is why it has a great potential for therapeutic uses in tissue regeneration and repair [1].

Stem cell research is an exciting field with great potential for heart regeneration [2]. The use of stem cells to allow cardiovascular diseases has rapidly progressed by proposing the idea of moderate improvement in heart function by reversing the myocardial injuries [3]. Transplanting stem cells can be achieved through several routes of delivery to the heart including intracoronary, intramyocardial, intravenous, and epicardial [4]. Each delivery has its own advantages and disadvantages with several factors to consider [3]. In the review by Sheng et al.,[5] they present their belief that cell delivery methods should be personalized based on the case rather than its overall success. They validate their statement by the example of a patient with an obstructed coronary artery with an ischemic myocardial injury. When selecting a cell delivery method, intracoronary may not be the most favorable solution regardless of its overall effectiveness in other cases. This reveals that there may not be an overall preferred method of delivery but there are preferred deliveries based on each case individually [3]. Although cell delivery modalities are important, the cell type, dose, and timing of administration are also important to discuss for cardiac regeneration. This is shown in Figure 1.

Stem cell research has been rapidly progressing from the use of animal model systems of human disease which has led to many of the clinical studies to date [4]. Researchers have now used species hearts and injected stem cells such as MSCs, ESCs etc., into the species, resulting in some functional cardiac improvement. Large and small animal models have been used and have shown the different mechanisms and types of stem cells to heal the injured myocardium, helping to lay the

${ }^{\star}$ Correspondence to: Vincent S Gallicchio, Department of Biological Sciences College of Science Clemson, University Clemson, SC 29637, USA, E-mail: vsgall@clemson.edu

Key words: congenital heart defects; congenital; stem cells; prevalence, cardiac repair

Received: April 01, 2019; Accepted: April 17, 2019; Published: April 25, 2019 
groundwork of clinical application [7]. According to Collins and Russell [6], the key elements for stem cell therapy for cardiac repair are the type of cells and the mechanism by which the cell can improve heart function [6]. The idea of using stem cells for regenerating damaged myocardium is a topic of intense research and the goal is to direct this continuous advancement and conduct more experiments so that it can be used for the treatment of chronic human diseases, such as cancer, neurological, diabetes, and cardiovascular [8]. By using stem cell therapy, the goal is to promote the engraftment of new, beating cardiomyocytes into where the heart needs it [6]. There are a small but growing number amount of research for stem cell therapy for children with congenital heart disease [7]. Stem cell therapy studies also show the ability for them to improve angiogenesis and mitigate oxidative stress in the setting of pressure overload. These stem cells could prevent right ventricle failure under pressure overload in specific forms of CHD [9].

Congenital heart disease is defined, according to Avolio and colleagues [10], as an "abnormality in heart structure that occurs before birth, while the fetus is developing" [8]. As stated before, congenital heart disease affects 1 in 100 babies every year in the United States [7] and twenty five percent of these infants will require surgery within their first year [11]. The timing of surgeries varies depending on the severity of the defect of each patient, but some patients may need surgical intervention within days or weeks from birth [4]. Children with this defect go through numerous surgeries throughout their lifetime which can be highly invasive and places a significant burden on the lives of these children and their families by having physical, psychological and economic impacts [8]. Physical impact includes the limitation of exercise as the patients are put at a higher risk for activities they can or cannot do, but this varies from patient to patient. The psychological impacts include the child not growing up in a normal atmosphere as other children their age because of the numerous hospital visits and doctor appointments. This can hinder their social abilities because some patients have to be excused from a vast amount of school for surgeries, hospital visits, etc. [4]. For example, patients with a specific congenital heart defect called Tetralogy of Fallot undergo surgery once at 3 to 6 months of age but may additionally need pulmonary valve replacement during the course of their lives [4]. Patients with congenital heart defects also may feel excluded because of their defect and uncomfortable with the scars from their open-heart surgery. Some examples of economic impacts include the high costs of treatment and the challenges families may have to go through in scheduling around work for hospital visits which can cause a huge strain on their daily lives. However, these impacts do vary depending on numerous factors including family's current situations and lifestyles [12]. The goal of stem cell therapy is to provide a treatment option with minimal invasiveness which will regenerate the myocardium that was lost or destroyed. Stem cell therapy is not limited to only improving myocardial function or decreasing scar formation but also has been demonstrated to provide a safe and feasible option in adult patients with heart failure. The clinical trials in adult heart disease is shown in Table 1. However, it has not been achieved yet, it is believed that the goal is to be reached soon by using these therapies with the ongoing clinical trials [13]. Because of the benefits in preclinical studies, there is growing evidence supporting stem cell therapy for children with CHD especially those with single ventricle physiology [7].

The heart is the least regenerative organ in mammals which makes it difficult to treat. In patients that do not respond to current medical therapies, stem cell therapy is shown to be an exciting option. The growing discoveries that have been made recently on this exciting field have improved the understanding of cardiac development and stem cells capabilities to treat a wide array of degenerative diseases. The advancement of the use of induced pluripotent stem cells has showed to direct cardiac reprogramming by factors crucial to the heart. The growing investigation of bone marrow stem cells and cardiac progenitor cells have had great success in preclinical and clinical studies. These advances help pave way to treat children with CHD by regenerative medicine [14]. Furthermore, by delivering stem cells into the injured region of the heart, the goal is to regenerate new myocardium, restore

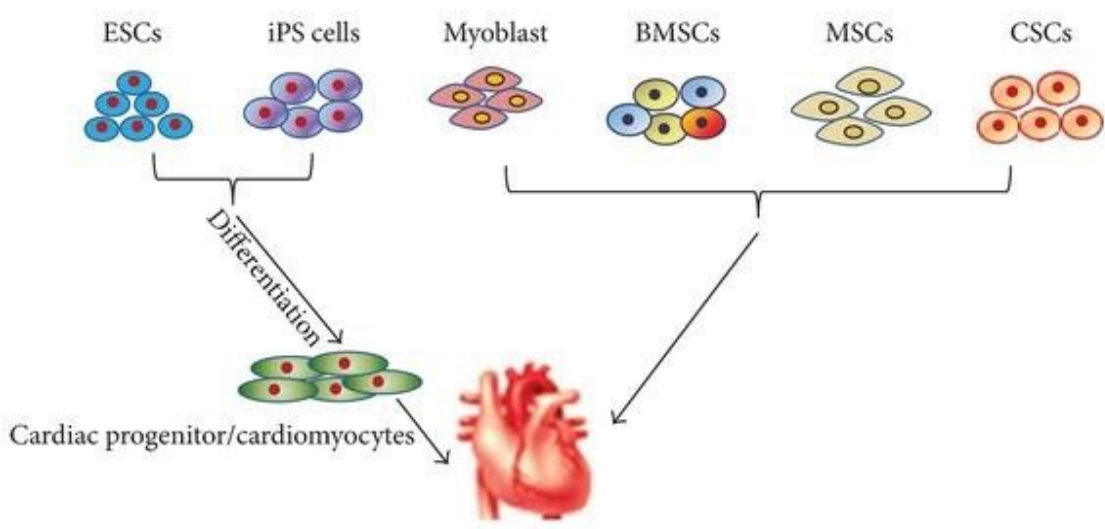

Figure 1. The types of stem cells for cell-therapies is important to note. Embryonic Stem Cells (ESCs) and Induced Pluripotent Stem Cells (iPSCs) are both pluripotent stem cells that differentiate into cardiac progenitor cells that can be utilized for cardiac repair. On the other hand, myoblast, bone marrow stem cells, mesenchymal stem cells, and cardiac stem cells are multipotent and are mostly used to restore the heart function directly. It is vital to note the differences between the stem cells to effectively choose which ones to investigate for cardiac repair [5]

Table 1. Shown above are the ongoing clinical trials for stem cell therapy in adult heart diseases. This shows that many studies have been conducted for adult heart diseases, paving way for the therapy to be conducted in the pediatric population [14]

\begin{tabular}{|c|c|c|c|c|c|c|c|}
\hline Trial name & Sample size & Masking & Disease & Cell source & Cell type & Phase & NCT ID \\
\hline ALLSTAR & 134 & Double blind & IHD & Allogeneic & CDCs & Phase II & NCT01458405 \\
\hline DYNAMIC & 42 & Double blind & DCM and IHD & Allogeneic & CDCs & Phase I & NCT02293603 \\
\hline CONCERT- HF & 144 & Double blind & IHD & Autologous & $\mathrm{CSCs}+\mathrm{MSCs}$ & Phase II & NCT02501811 \\
\hline TAC-HFT II & 55 & Single blind & IHD & Autologous & CSCs + MSCs & Phase I/II & NCT02503280 \\
\hline
\end{tabular}


blood flow, and improve contractility [15]. In this review, the effects and advancements of stem cell therapy to potentially treat congenital heart disease is discussed.

\section{Current treatment for congenital heart defects}

Current surgery consists of implanting synthetic or biological valves, stents, or patches that allow the patients to survive, but limitations include risks of rejection, thrombosis, and inflammation as well as the inability for the implant to grow with the child, which results in more treatments [11]. These implanted devices are effective in the short term, but the long-term approaches are poor requiring replacement within 3 to 8 years depending on the size and growth of the patient and how they respond [8]. Numerous other surgeries include the implantation of these materials which are expected to be close to the native cardiac tissue of the patient [12].

Robert Gross was the first to establish pediatric cardiac surgery in 1938. Open heart surgery on cardiopulmonary bypass became established in the 1950s. Since these prominent events and advancements, CHD has been reduced tremendously. However, more complex forms of this disease still remain and cannot be treated but slows down the progression of the disease. Over time, the patients will be at risk for heart failure and require heart transplantation. The heart has limited ability to proliferate cardiomyocytes and regenerate the myocardium [16]. Therefore, surgery remains the most established treatment because of its effectiveness and advancement [4].

Still, there has been a shift of attention to stem-cell therapy as an alternative treatment option because of stem cells potential for cardiac regenerative medicine [17]. This interest also spring from the advancements and emergence of stem cell therapy in adult patients with ischemic heart disease and if those stem cells could work for patients with CHD [13]. From experience with Ischemic Heart disease, the idea is to build upon those trials to translate them for CHD. Two promising clinical trials such as BOOST trial (bone marrow transfer to enhance ST elevation infarct regeneration) and REPAIR-AMI (the reinfusion of enriched progenitor cells and infarct remodeling in acute myocardial infarction) showed an increase in the function of the left ventricle with adult patients with ischemic heart disease. After these two trials, many clinical trials were attempted but they failed to report the improvements as seen before. These discrepancies are not clear but may be due to the percentage of cell surface markers $\mathrm{CD} 34^{+}$and $\mathrm{CD} 1333^{+}$or the impact of aging [18].

Hypoplastic left heart syndrome (HLHS) and tricuspid atresia are more severe conditions that require many procedures and usually result in heart transplantation. Only $30 \%$ of the patients with this condition stayed alive by the age of 16 years in 1984 . Children with many types of single ventricle heart defects normally require three surgeries, including the Norwood, Glenn and Fontan procedure to assist the patient in the growth and survival of life. However, after the last stage, the Fontan procedure, the next step is normally heart transplantation due to volume overload [7]. Volume overload is when there is too much blood in one of the heart chambers for the heart to function correctly. Heart transplantation is the last therapeutic treatment option when other forms of surgery are no longer an option since the heart is a limited organ with minuscule capacity for repair [19]. Heart transplantation occurs as a treatment usually when these patients have tried medications or other surgeries, but their conditions have not improved. Not only are heart transplants a costly procedure, but it becomes difficult to achieve due to severe donor shortages [7]. Heart transplantation is invasive, costly, and excludes patients who are not candidates for transplantation given their comorbidities. Because of the increasing number of patients with heart failure and children with $\mathrm{CHD}$, there are not enough organs for transplantion [20]. After trying numerous surgeries and medications that do not improve the heart functionality, transplantation is left as the only option, which includes complications. As stated before, complications include shortages of donors and viable matches as well as the risk of the donor's heart rejecting the recipient. Stem cell therapy could become a potential option to this which is why research has become recently highly anticipated. This stems further research for stem cell therapy and the idea of an alternative option to avoid heart transplantation [21]. The three consecutive surgeries: Norwood, Glenn and Fontan, are performed within the first few weeks of birth, the second between 4 to 6 months and the third within the first 4 years. The first two stages are displayed in Figure 2. Stem cell therapy could decrease the need for the load of surgeries within the first few years of life [7].

Furthermore, surgery has allowed patients to survive and thrive from CHD. However, these procedures place pressure and volumes loads on the heart which can over time lead to heart failure and require more surgery. There is limited therapeutic options for surgery available because of the heart's low capacity to form new cardiomyocytes. A deeper understanding for stem cell therapy for the treatment of CHD could transform the treatment of CHD and other cardiac diseases [16].

\section{Potential for cardiac repair}

After heart surgery, children with Congenital Heart Disease (CHD) begin a healing process. This natural healing process is a mechanism of regeneration that recent evidence has shown, resembling stem cell mobilization. This realization gave rise to the idea of stem cell therapy. For preclinical proof-of-concept studies in congenital heart disease, many types of stem cells have been applied in pressure-overload right heart models. Initially, direct myoblast transplantation in an ovine model did not show a functional benefit however some cells migrated to the right myocardium. Later, the development of sheet technology enabled an increase in cell engraftment with increased ventricular function and decreased cardiac fibrosis. New advances include the safety and feasibility of intramyocardial delivery of umbilical cord blood stem cells in large animal models [22].

One report demonstrated the collection of the drain fluid in CHD patients after surgery, which contained the immunofluorescence staining of CD117 (c-kit) that would reveal the presence of cardiac stem cells. C-kit is a stem cell marker for cardiac stem cells. It is the most commonly used since c-kit positive cells have been shown to differentiate into cardiac cells including cardiomyocytes, vascular

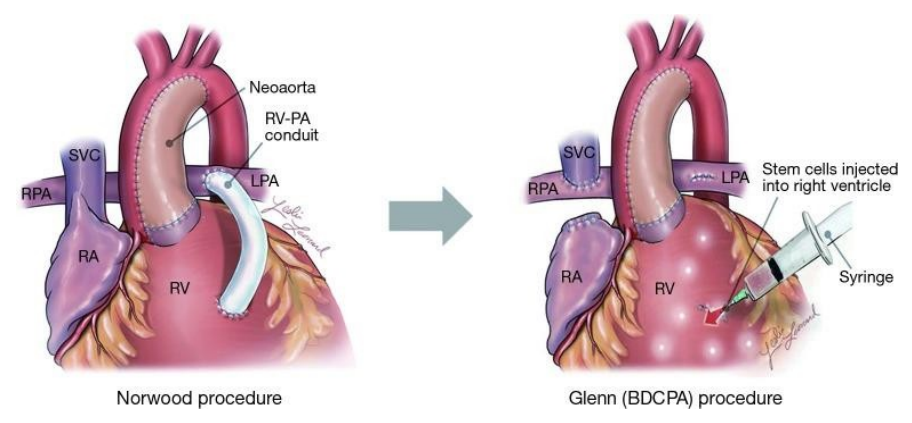

Figure 2. Stage 1 of the three-stage heart construction is known as the Norwood operation. Following this stage comes the second called the Glenn operation. These are both for the reconstruction for patients with Hypoplastic Left Heart Syndrome (HLHS). With the current trials of stem cell therapy for this defect, the cells are administered after the stage II, Glenn operation and sometimes the Stage III Fontan procedure that is not shown in the figure [7] 
smooth muscle cells, and endothelial cells. In this report, it is stated that these cells can reduce the size of myocardial infarction scars as well as maintain the left ventricle ejection function in vivo. This was completed by isolation and expansion of the stem cells by using flow cytometry. The purpose of the study was to provide a practical method for obtaining autologous human stem cells which was successfully done by using the stated method [23]. Another similar study by Li et al. [24] showed that both c-kit- and c-kit+ treatment improved heart function. Their results demonstrated higher angiogenesis and anti-apoptotic effects which results in better recovery of heart function.

Using stem cell therapy for cardiac regeneration is quite the challenge in figuring out which cell source is the ideal candidate. There have been many published studies on the effects of cell therapy for all cardiac diseases. These studies include cell types examined in animal models that includes bone marrow stem cells (BMSCs), skeletal myoblasts, human umbilical cord blood cells (hUCBSc), cardiac stem cells (CSCs), Mesenchymal stem cells (MSCs), induced pluripotent stem cells (IPSCs) etc. [25]. It is important to base the stem cells on the origin of them, i.e., bone marrow, resident cardiac cell etc., and their ability to differentiate [19]. According to Tsilimigras [4], delivery can consist of origin options such as intramyocardial, intracoronary, intravenous, and epicardial. Differentiation options depend at which the stem cells are harvested which give them different properties. Over the past decade, there has been remarkable stem cell advancement and popularity to the field of cardiology [19].

Researchers have used animal hearts to experiment the improvements of stem cells in cardiac disease. Initial animal experiments had used skeletal myoblasts and found improved heart function, but later these showed that the native heart cells did not couple with the new cells, thus resulting in ventricular arrhythmias. However, this experiment ignited the idea to use other cells to increase the longevity of cardiac function. For example, more recently the stem cells isolated from bone marrow, amniotic fluid, etc., have been used [6]. Since this disease occurs in the early stage of development, cardiac regeneration in the pediatric setting is measurable and potentially modifiable. This modification of cells to promote cardiac regeneration has demonstrated significant improvement in cases. Therefore, focusing on this cardiac regeneration could give new insights in the treatment of this disease. This could transform the management of CHD and be cost-effective [26].

Placental stem cells are a growing interest for the potential of multipotency and since they are easily accessible. Stem cells derived from the placenta have been investigated in a number of disease processes including wound healing, ischemic heart disease, and congenital defects. The use of stem cell therapy for congenital defects is of interest because of the immunologic immaturity and immunotolerant environment of the developing fetus. More research needs to be conducted; however, placental stem cells are a promising therapeutic agent for congenital diseases in the fetus [27].

The best responders to cell therapy may be children. This is because their myocardium is inherently more pliable and will respond more efficiently to the transplanted cells. This is supported with the Transcoronary Infusion of Cardiac Progenitor Cells in patients with single ventricle physiology (TICAP) and Cardiac Progenitor Cell Infusion to Treat Univentricular Heart Disease (PERSEUS) trials that demonstrated the best response may occur in infants of the smallest size, youngest age, and lowest ejection fraction [28]. According to a report in 2016 [29], twenty reported pediatric patients had received a stem cell therapy product for CHD or end-stage cardiomyopathy that are discussed in this review. Research on stem cells has rapidly grown in the past decade and holds impressive potential to be a therapeutic option for some diseases.

However, only few of the laboratory researchers have successfully transited to the clinical trial phase [30]. In 2012, an article reported on the evolving field of stem cell therapy. During that time period, stem cell therapy had only focused on cardiomyoplastly for heart muscle disease with clinical trials published. They reported the support for the potential role in stem cell therapy for congenital heart disease and the role of them to create cellular grafts and structures using bioengineering technology. Great improvements and advancements of stem cell use in congenital heart disease has been displayed and conducted since this article was published. This shows the rapidly progression of stem cell therapy and its use to treat diseases and injury [31].

This review will discuss how stem cell therapy is being used in the management of congenital heart diseases.

\section{Cardiac progenitor cells}

Cardiac progenitor cells (CPCs) are endogenous cardiac stem cells that are found to express stem-cell like features in the adult heart. They are found to contribute to the regeneration of cardiac tissue after injury. These cells restorative potential and differential capacity into a variety of cells types of cardiac tissue have gave way to using them to treat cardiac injuries [32].

Through the use of resident human cardiac progenitor cells in stem cell therapy, it lays promising alternative options for treatment for patients with heart injury. However, these cells are rare in the human heart. That is why finding an efficient source and proper surface marker for the isolation of these cells would help make these more easily accessible. This study found the presence of CD133 that expressed cardiogenic stem-like cardiac precursors in patients with CHD hearts. This finding would help make it easier for the stem cells to be isolated and stored for future use in patients with CHD [33].

Through the discovery of a subpopulation of c-kit+ cardiac stem cells, the mammalian heart is now shown to regenerate following myocardial injury. One report showed the isolation of c-kit+ human cardiac stem cells (hCSCs) and tested them by injecting the cells into mice and rats. They found that these cells differentiated into cardiomyocytes thus improving the functionality of the injured hearts. The human myocardium structurally and functionally integrated with the rodent's and increased heart performance. This was performed in vitro and showed these stem cells can be used further for patients with heart failure [34].

Another study determined the role of cardiac stem cells (CSCs) in children with end- stage heart failure (ESHF). They completed this by taking tissue samples from explanted heart of children undergoing heart transplantation with ESHF and from an age-matched CHD patient with normal myocardium. The results showed that the ESHF myocardium had an increased expression of c-kit+ CSCs compared to the CHD patient's myocardium. In addition, the ESHF patients had a significantly reduced telomere length as well which suggest a diminished functional capability in those cells. However, the reduction of telomere length may mean that the cells have diminished in their functional capacity. Further studies need to be conducted to further characterize the functionality of these CSCs in patients [35].

Clinical trials are ongoing for the treatment of heart diseases such as HLHS. In HLHS, there is a single ventricle defect. In 2015, patients 
with HLHS were given autologous cardiosphere-derived cells (CDCs) by intracoronary administration. The patients right ventricular ejection fraction remained persistently improved during the 36-month followup [36]. As mentioned above, this trial was the transcoronary infusion of cardiac progenitor cells (TICAP) which was designated for patients with HLHS. The study's goal was to determine the safety and clinical outcomes of cardiosphere-derived infusion after staged palliation. These stages included the second or third stage which are the Glenn and Norwood operations, respectively. Fourteen patients with HLHS received the cells after their surgery as well as a control group. After 36 months, no complications were found, and the echocardiogram showed significantly greater improvement in the right ventricular ejection fraction (RVEF) compared to the control group. This study supported the idea that stem cell therapy can help reduce heart failure and enhance the functionality of the damaged heart in patients with HLHS [37].

Next, the Cardiac Progenitor Cell Infusion to Treat Univentricular Heart Disease (PERSEUS) trail was conducted to follow-up on the TICAP trial. CDCs were administered through the coronary arteries after either the Glenn or the Norwood procedure. The group treated with CDCs showed an improvement in right ventricle function at three months compared to the control group. At 36 months, there was an improvement in the right ventricle ejection fraction in the patients receiving CDCs. The TICAP and PRESEUS both reinforced the clinical benefits of CDCs and showed their consistency and safety [38].

Further characterization of cardiac progenitor cells is needed to provide further understanding in the dynamics of specific CHD. In addition, further understanding is necessary for developing therapy for heart failure [39].

\section{Embryonic stem cells}

Embryonic stem cells (ESCs) are pluripotent, this means that they can give rise to all cell types except the placenta and umbilical cord. They are undifferentiated cells that are derived from preimplantation-stage embryos. They have the capability of dividing without differentiating for a prolonged period in culture and are known to develop into cells and tissues of the three primary germ layers. Because of this, they are valuable since they provide a renewable resource for studying normal development and disease [40]. Human embryonic stem cells (hESC) have raised ideas on alleviating disease and injury. Their pluripotency and capacity for self-renewal ignite research to identify mechanisms to differentiate these cells to specific cell types that will treat diseases or injuries. However, there is controversy surrounding these stem cells regarding the ethical issues [41]. These ethical issues have delayed the initiation of clinical trials in patients with heart diseases. This is why induced pluripotent stem cells may be a better option. IPSCs are autologous and are defined as an adult stem cell that has been genetically altered to behave more like an embryonic stem cells. This means that they are also pluripotent and can form into all cell types [15].

Therapeutic options are limited especially for patients with right ventricular failure with congenital heart disease. A report used a pig with overloaded right ventricle dysfunction to assess the feasibility and effects of cell therapy. They believed that human embryonic stem cellderived cardiac progenitors provided a good idea to treat this disease because of its proliferation capacity. They administered human $\mathrm{MesP}^{+} /$ SSEA- $1^{+}$cardiogenic mesodermal cells and observed their effects three months later. The results concluded that all the large animals survived without complication thus leading to cell therapy being harmless. The treated group showed arrhythmic susceptibility and beneficial effects on fibrosis [42].

\section{Amniotic fluid-derived stem cells}

Amniotic fluid contains a rich source of stem cells that can be used in treatment of diseases [43]. This fluid fills the amniotic cavity and serves as protection for the fetus as well as allowing the fetus to move [44]. The amniotic fluid is routinely discarded after birth, which reveals the easy accessibility of the amniocytes [10] Since the fluid can be collected easily with minimal risk, it shows a greater advantage in collecting stem cells such as mesenchymal stem cell and induced pluripotent stem cells [44,45]. Amniotic fluid-derived stem cells (AFSC) have been explored as an experimental approach for improving children with CHD as well as other congenital anomalies [46]. Since the amniocytes are easily accessible by collecting them from the amniotic fluid, the process is recommended for an evaluation for many fetal anomalies because of its convenience [46]. This convenience or advantage of using amniotic fluid as a stem cell source includes the relative ease of harvesting autologous fetal stem cells [47]. There would be no need to wait until delivery for the cell harvesting since it could be collected during diagnosis [43]. Amniotic fluid derived stem cells, originating from the fetus itself, can easily be expanded in vitro and ready to use as an autologous therapy [46].

Amniotic-fluid derived stem cells have shown the capability to differentiate into cardiomyocytes. Kunisaki [46] states, "an alternative, regenerative medicine-based approach to conventional therapies would be to generate autologous replacement cardiomyocytes specific to each patient. Studies have shown the idea of tissue-engineered patches could be made from living functional cells that can grow along with the patient as they get older instead of the current device use of synthetic patches and fixed grafts which run the risk of potentially requiring further surgeries. When a patient receives a synthetic patch or fixed graft, these devices are unable to grow with the patient and thus will require further surgeries. The functional tissue engineered patch that would contain embedded stem cells, would allow for growth and remodeling, since it would contain cardiac cells that match with native tissue, and thus would restore cardiac function. The patch would contain cardiac progenitor cells that are derived from the patient's own stem cells [10]. For example, amniotic-fluid based-stem cell technologies have been investigated with the fabrication of tissue-engineered heart valves. In another report, Schmidt et al.,[48] successfully used amniotic fluid as a single cell source enabling the prenatal fabrication of heart valves ready to use at birth [46]. In one study, AFSC was used to create tissueengineered heart valve leafless by isolating the stem cells from the amniotic fluid. The results showed the construct covered with AFSC and it remained a functional valve for 28 days of in vitro culture. Therefore, AFSC has shown its advantages as an effective cell source for a fabricated engineered heart valve thus showing the potential to be used for further implications [44].

\section{Mesenchymal stem cells}

Mesenchymal stem cells are defined as non-blood adult stem cells from a variety of tissues. However, it is not clear that mesenchymal stem cells from different tissues are the same. These stem cells are a population of pluripotent cells derived from bone marrow stromal cells.

Since they are pluripotent, they can differentiate into all cell types, this includes bone, cartilage, muscle, tendon, ligament, and adipose tissue. The unique characteristics of these stem cells have allowed in vivo evaluation of both autologous and allogenic MSC preparations which could promote cardiac remodeling after injury in animal models. According to the article published in July 2018, there had been 32 clinical trials with the use of MSCs to treat a variety of adult heart conditions [49]. 
Therefore, mesenchymal stem cells (MSCs) have been considered for stem cell therapy for Congenital Heart Disease in hopes of repairing and regenerating the myocardium thus improving cardiac function [10]. Mesenchymal stem cells were tested for their safety of administration in Weiss, et al. [50] report regarding patients with COPD. This study concluded that systemic administration of MSC appeared to be safe which provided support for further investigation on the use of cell therapy for other heart diseases [15]. Adult mesenchymal stem cells are considered effective for improving cardiac function and are immune-incompetent [51]. These stem cells are available following isolation, pluripotency, and differentiation capacity [10]. For example, bone marrow contains a great source of mesenchymal cells, which has been used in clinical studies since these stem cells can be easily isolated. The mechanism by which they are isolated can be by using magnetic columns or flow cytometry to isolate the cell of interest, and in this case, mesenchymal stem cells. These stem cells have the ability to promote myocardial angiogenesis to improve ventricular function. The use of MSCs from bone marrow mononuclear cells (BMNCs) has given informative data related to people will heart defects. However, mesenchymal stem cells are multipotent which means they can develop into more than one cell type. This runs the risk that they could differentiate into a variety of cell types when transplanted into the heart like bone, cartilage, and adipose tissue which would thus form in the heart [15].

Umbilical cord is full of stem cells and represent good therapeutic option since it comes from the fetus and the cells are pure and optimal [15]. When the mesenchymal stem cells are extracted, these stem cells are able to differentiate into classical MSC lineages (bone cartilage, and fat) as well as endothelial cells and cardiomyocyte-like cells $[7,15]$ The mesenchymal stem cells can be extracted from the Wharton's jelly which is a substance within the umbilical cord [15]. In preclinical studies, these stem cells have shown to improve left and right ventricular structure and function [7].

Studies of stem cells in animals have shown the ability to show that MSCs expresses cardiac lineage proteins such as cardiac troponin and GATA4 when injected in cardiac infarct models and improve cardiac function in those models [52]. Therefore, the ability to stimulate vascular and cardiomyocyte regeneration, which may positively influence cardiac metabolism and contractility [10]. Wehman, et al. [11] was the first to study the use of MSCs for stem cell therapy to improve right ventricle functionality. They evaluated placental mesenchymal stem cells (pMSCs) for their differentiation capacity into cardiomyocytes. By using these stem cells, they could potentially be used for cardiac repair and regeneration. By comparing protein expression before and after the stem cells were treated with cardiomyocyte differentiation media. Results showed the enhancement of pMSC cardiac protein content from the differentiation media treatment, but no production of beating cells. Conversely, they also found the undifferentiated pMSCs improved proliferation of a cardiac cell population, which shows the idea of using these stem cells as surgical implants for cardiac regeneration and repair should be further researched [11].

Furthermore, mesenchymal stem cells are mesoderm-derived stem cells that can be isolated from various organs as stated before. Human MSCs were used as a therapeutic strategy to treat a porcine model of pressure overload in the neonatal right ventricle that is discussed later. This is correlating with the fact that intramyocardial delivery of hMSCs have been shown to preserve global and regional cardiac functions, attenuate remodeling, and stimulate endogenous progenitor cell proliferation to promote neovascular formation and myocyte cycling.
MSCs secrete growth factors including growth differentiation factor 15 and it is noted that it could reverse right ventricular hypertrophy [22].

\section{Preclinical studies using mesenchymal stem cells}

A report shows the stem cell therapy for myocardial repair in a rodent where bone marrow cells when injected in the infarcted area of the heart. This experiment observed in the treated mice, there was $68 \%$ of new cells in the infarcted heart and $40 \%$ of the heart tissue was regenerated. This new heart tissue was comprised of proliferating myofibroblasts and vascular structures. However, other researchers have been unable to attain these same results which has sprung many questions about this early study but has helped lead further research6. Results of some studies suggest these stem cells from bone marrow can differentiate into cardiac myocytes while results of others do not. It has been effectively shown in animals that MSCs can differentiate into cardiac myocytes but there is no evidence in human clinical trials. Since MSCs are easy to grow, they have also been shown to differentiate into cardiomyocytes in vitro after exposure to 5-azacytidine7. This was shown in pig or sheep hearts after injecting mesenchymal stem cells and observing long-term engraftment, reduce scar formation, and improved cardiac function however, there is little evidence this works in vivo (Figure 3).

In addition, a study used a 4-month-old sheep that had a patch placed on its induced right ventricle overload. Umbilical cord blood, a source MSCs was intramyocardially injected into the right ventricle free wall. After three months, the sheep exhibited increased function of the right ventricle diastolic compared to the control sheep. However, in another model that was a 1-week- old sheep with the same right ventricle pressure overload but by implicating the main pulmonary artery banding. They injected human cord blood stem cells into the right ventricle myocardium and the model demonstrated improved function as well [7].

Porcine models were also successful in the use of pulmonary artery banding with the improvement of induced right ventricle function. They intramyocardial injected 1 million human MSCs and concluded that MSC-treated pigs showed less right ventricle dilation and improved right ventricle function compared to the controls. Furthermore, this experiment with the RV pig model showed a greater expression of growth differentiation factor 15 (GDF-15) in the myocardium that has also been shown to inhibit hypertrophy. This conclusion stems the idea that stem cells interact with their environment to promote favorable
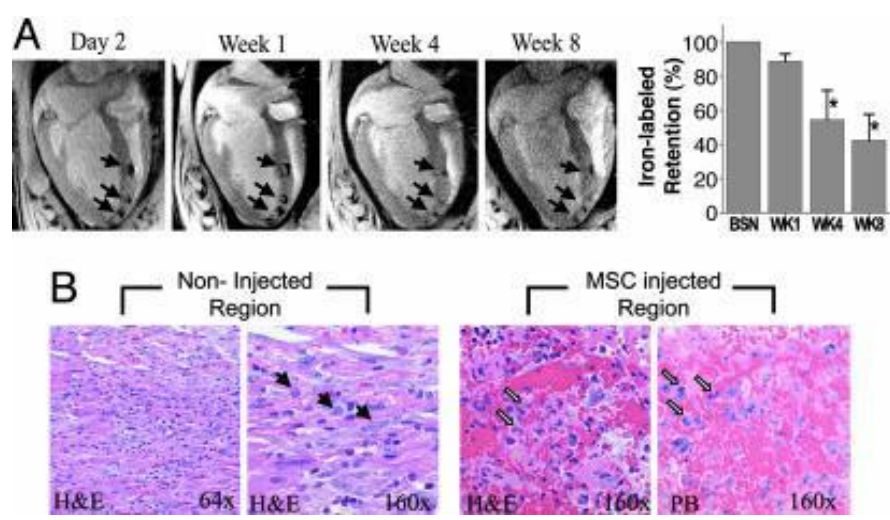

Figure 3. This represents allogenic porcine MSCs from an MRI. In the section labeled "A" there is imaging obtained after the injection of the stem cells. This shows the presence of MSCs after injection in all weeks afterward. The bar graph represents Feridex retention over an 8-week course. The section labeled "B" shows histologic observation of the postinfarct myocardium comparing the non-injected and MSC injected regions [53] 
ventricle remodeling and not on differentiation itself [7]. In addition, Wehman et al. [11] was the first to study the use of MSCs for stem cell therapy to improve right ventricle functionality by using a porcine model. Not only did they want to test the idea that MSCs preserved right ventricle function but also to attenuate ventricular remodeling. They did this by using a neonatal swine model of right ventricle (RV) pressure overload [3]. Stem cell therapy mechanisms in these animal models have yielded important insights of stem cell therapy in congenital heart defects in humans since there are a small, but growing amount of clinical trials for those patient's [7].

During pediatric cardiac surgery part of the thymus is removed and found to be a good source of MSCs and possess regenerative properties, according to Wang et al. [54]. They found that the properties of neonatal thymus MSCs (ntMSCs) also expressed great proangiogenic factors thus having the ability to promote cardiac function. Their study consisted of applying ntMSCs to the left ventricle (LV) epicardium of rats immediately after left coronary ligation. Their results comprised of improved LV function, vascular density, and decreased scar size showing that ntMSCs have cardiac regenerative properties.

Another study found that MSCs have a great effect on cardiac cKit+ cells (CSCs) and their migration, proliferation, and differentiation. This finding suggests that by using MSCs and MSCs through stem cell therapy could provide a mechanism to repair the damaged cardiac tissue. They proved this through using the postnatal murine heart and showed that the resident $\mathrm{cKit}+$ cells proliferate, migrate, and form cardiomyocytes. When the CSCs were combined with MSCs, the CSCs proliferated. This means that the MSCs enhanced CSC proliferation through the stem cell factor/cKit pathways [55].

\section{Clinical application of mesenchymal stem cells}

Mesenchymal stem cells have been shown clinically in early phase trials for their safety. Feasibility, and preliminary efficacy in improving regional contractility and quality of life. Since mesenchymal stem cells have been verified extensively in preclinical animal models, the breakthroughs for clinical trials are increasing. The initial clinical trial demonstrated patients after an acute myocardial infarction be administered mesenchymal stem cells. The results showed no immune response and the MSCs promoted improvements in pulmonary function and left ventricular function. In addition, there was the POSEIDON trial (the percutaneous stem cell injection delivery effects on neomyogensis) that was for patients with chronic ischemic cardiomyopathy. These patients were administered allogeneic and autologous mesenchymal stem cells and compared the two cells. They found the allogeneic MSCs caused no relative increase in serious events and did not stimulate an immune reaction. However, both of these stem cells reduced the size of the infarct by about $33 \%$ and improved the patient's quality of life [18]. This clinical trial paved way for the following and could be used as a template for other stem cell therapies for other heart diseases. Studies have shown clinical trials of stem cell therapy with adult patients with congenital heart disease conveying results of left ventricle function improvement [17]. However, only a few cases have been reported for infants.

One study included an 11-month-infant possessing hypoplastic left heart syndrome in a study recorded by Rupp, et al. [56]. The patient was in critical condition after undergoing cardiogenic shock. Treatment included autologous bone marrow cells that were transplanted back to the patient by intracoronary bolus injection. A year after stem cell therapy, the patients critical condition improved tremendously with improvement in his brain natriuretic peptide and his right ventricle ejection fraction. The report stated no complications during cell infusion [57]. At 3-months follow-up the report states improved cardiac function and a reduction of end-diastolic and end- systolic volumes [58]. A similar increase in these conditions was reported in a nine-year-old girl with congestive heart failure after an anterior myocardial infarction. After undergoing initial treatment, this patient can back to the hospital a year later with a significant drop in ejection fraction. She was successfully treated with autologous bone-marrow derived progenitor cells via transcoronary injection to the left anterior descending artery. At a twelve-week follow up, the patient was reported with remarkable improvement of her symptoms and the ejection fraction [18].

In a clinical trial, using umbilical cord mesenchymal stem cells in adults with chronic heart failure reduced the ejection fraction. Their treatment was randomized to intravenous infusion of these stem cells or placebo [7]. It was concluded that there were improvements in the function of the left ventricle and quality of life. This study concluded the safety of intravenous infusion of UC-MSC for patients with heart failure which gave important information for further studies regarding cell therapy in heart disease [59].

\section{Induced pluripotent stem cells}

Induced Pluripotent Stem Cells (iPSCs) are adult cells that have been reprogrammed back to an embryonic-like state for the purpose of developing an unlimited source of any type of human cell needed [47]. Stem cells comprise of two major classes including pluripotent, which means they can become any cell in the human body [60]. Induced Pluripotent Stem Cells (iPSCs) have the potential to supply newly born myocytes that can regenerate damaged myocardium [61]. iPSCs can differentiate into several types of cells including cardiomyocytes as well as have the capacity of self-renewal [62]. These stem cells can be readily generated from the somatic tissues of patients [63]. These stem cells are obtained from the ex vivo reprogramming of fetal or adult somatic cells, like fibroblast. They make a great choice for application in regenerative medicine because of these properties of being able to differentiate in cells of the three embryonic germ layers and thus having the capacity to differentiate into most of the human body cells. In addition, these stem cells can differentiate embryonic stem cells toward cardiomyocytes which then can provide a great potential for cell therapy. It can also serve as in vitro models of CHD for the study of underlying mechanisms, drug screening, and safety test [63]. Therefore, for Congenital Heart Disease, iPSCs could be taken from the patient's cells and differentiated in vitro in vascular and myocardial cells before autologous transplantation [10].

A report investigated pluripotent characterization of reprogrammed amniotic fluid derived stem cells (AFSC) into induced pluripotent stem cells (iPSC) by mRNA transfection. This is because AFSC are multipotent and cannot directly differentiate into contractile cardiomyocytes (CM). However, iPSC can be differentiated into force-generating CM. Studies shows iPSCs can be generated from AFSC, thus being able to differentiate into functional CM. The study performed this without the use of viruses to show the AFSC-derived iPSCs are capable of cardiac differentiation. The results concluded to be accurate showing functional CM however, the implications included the cells being immature with delayed contraction, incomplete gap junction formation, and poor upregulation of Desmin. Furthermore, this was the first study to achieve those functional CM with non-viral reprogramming showing that AFSCs present an ideal source for stem cell therapy for cardiac regeneration and needs to be further researched to improve the implications of differentiation efficiency and cardiac maturation [43]. 
Human induced pluripotent stem cells (hiPSCs) offer a unique way to study the genetic mutations and developmental pathways associated with CHD. One report reveals using these stem cells as a model for heart development and CHD. These models help understand the development of CHD and explore novel therapies for the patients. The use of hiPSCs present an unprecedented opportunity to generate disease specific models to accomplish the goal of understanding unknown mechanisms of CHD as well as uncovering new therapeutic targets. These cells will provide a platform for preclinical trials of gene therapy and cell-based therapies with specific application for CHD [64].

The cardiovascular cells from patient specific iPSC contain gene mutations associated with the pathogenesis of CHD. There have been many studies to demonstrate the use of these stem cells to model CHD. According to a report published in 2016, many studies have been conducted regarding the application of hiPSCs for a variety of cardiovascular disorders such as HLHS and other congenital heart diseases [65].

With the invention of iPSCs, further therapeutic potential for cardiac regeneration has been produced. These stem cells can be induced to generate functional cardiomyocytes for regenerative cardiac therapy in patients. This ignited the findings of fibroblast converting directly into cardiomyocyte-like cells that could allow for cardiac repair. This report was documented in 2013 and stated how regenerative cardiac therapy with human pluripotent stem cells is moving closer to clinical testing. This shows the rapid progression and advancements this field has achieved [66].

\section{Preclinial studies using induced pluripotent stem cells}

In 2007, Yu et al. [62] reprogrammed human somatic cells into iPSCs by using four genes. These genes included Nanog, Oct4, Sox2, and Lib28. By using these four factors, they were able to reprogram the cells to generate iPSC lines and direct the cells to differentiation into CM. These stem cells showed the potential to differentiate into functional cardiomyocytes. This study allowed further investigation on the underlying mechanisms of heart diseases and provoking new therapeutic options [45].

Particularly, research has demonstrated the injection of cardiomyocyte- like cells generated from iPSCs on MI animal models and shown that the iPSCs are able to integrate with the cardiac tissue and thus improve cardiac function [21]. One report demonstrated the generation of iPS cell-derived cardiomyocyte sheets and proved that these cells exhibit contraction and relaxation in vivo with the myocardium [61]. This was accomplished by using a large animal model. iPS-derived cardiomyogenesis is ongoing and soon to be applied clinically for treating heart failure [61]. Similarly, Liu B, et al. [67] demonstrated extracellular vesicles secreted from cardiomyocytes derived from induced pluripotent stem cells provided contained great improvement in infarcted hearts. Extracellular vesicles decreased arrhythmic burden, decreased cardiomyocyte death and reduced the infarct size showing that this may help understand cardiac repair and regeneration [67].

\section{Clinical applications of IPSCS}

One congenital heart disease named Hypoplastic Left Heart Syndrome (HLHS) could be caused by intrinsic cardiomyocyte dysfunction. Any myocardial susceptibility component of this disease is yet undefined. So, to study the molecular mechanisms of this syndrome, iPSC lines were generated from HLHS patients and they were differentiated into cardiomyocytes. Observed in the HLHSiPSC-cardiomyocytes were alterations of gene expression profile. This included a downregulation of key cardiac markers MESP1 and cTnT. These cardiomyocytes also showed a lower ability to give rise to beating clusters compared to controlled cardiomyocytes. These HLHS-iPSC lines demonstrated developmental and functional defects that could compromise their ability to contribute to cardiogenesis in vivo. Moreover, the research on the use of iPSC to create in vitro models of cardiac disease have been limited due to gene mutations that compromise the functionality. However, this clarifies the analysis of the similar functioning cardiomyocytes generated from the HLHS-iPSC showing it is easy to show if they recapitulate the functional defects observed in the patient [63].

As stated earlier, studies in hiPSCs will help understand CHD (Figure 4). Two studies characterized hiPSC-derived CM from HLHS patients. One report showed cardiomyocyte differentiation and reduction of the expression of transcription factors in CMs derived from hiPSCs generated from these patients. These results were consistent with in vivo findings in midgestational fetuses with HLHS. The second report also showed decreased myofibrillar organization [64].

\section{Clinical study using umbilical-cord-derived stem cell}

Umbilical cord blood is used to isolate MSCs which are pluripotent and can differentiate into all cell types, including cardiomyocytes [49]. One study used a large animal model mimicking postsurgical scenario of Tetralogy of Fallot (TOF). They used umbilical cord blood mononuclear cells (UCMNC) and transplanted them intramyocardially. Their results showed the safety and feasibility of the treatment and believe through enhanced angiogenesis there was a positive influence in the diastolic properties of the right ventricle [68]. Preclinical studies mostly have focused on modeling right ventricle dysfunction of failure and pulmonary artery hypertension by using pressure or volume overload in vitro or in vivo [4]. Cell-based strategies to treat heart failure in adults have been reported but there is little information on these strategies for children with congenital heart disease [69]. Two

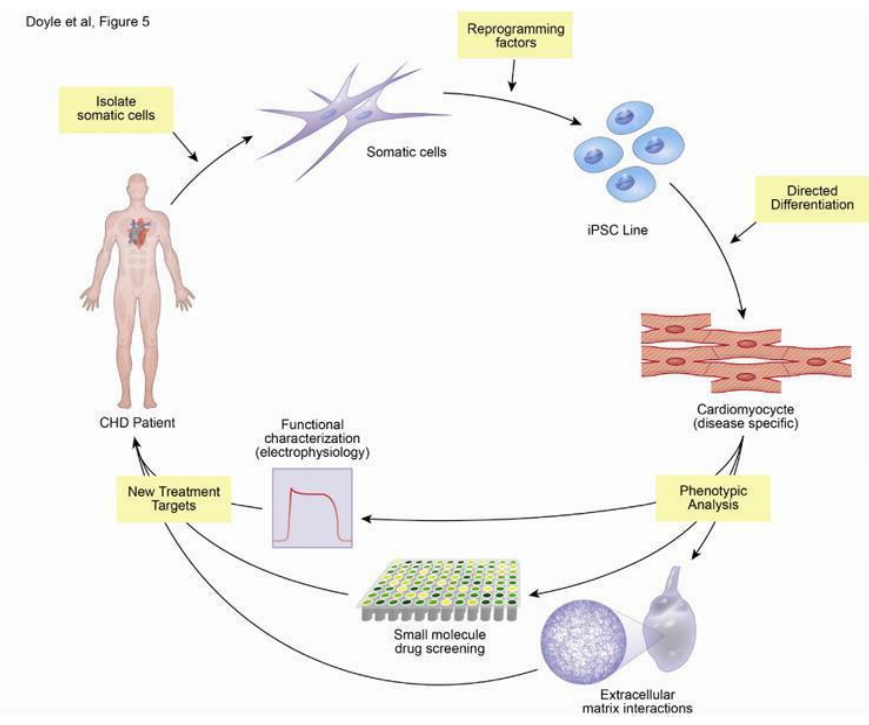

Figure 4. This diagram represents the process of isolating somatic cells from CHD patients, reprogramming the cells into using factors to generate an iPSC Line. Next, they are directed into differentiation into cardiomyocytes and phenotypically analyzed. This is to characterize the pathophysiology of the patient with CHD to further investigate the disease as well as therapeutic options [64] 
ongoing cases are being conducted for patients with hypoplastic left heart syndrome, a type of congenital heart disease. This disease is a complex and rare heart defect that happens at birth when the left side of the patient's heart is severely underdeveloped. In Minnesota at the Mayo Clinic's campus, there is a group of physicians and researched that aim to delay or prevent heart failure with patients with HLHS. This program, the Todd and Karen Wanek Family Program for Hypoplastic Left Heart Syndrome, conducts research that includes regenerative medicine. They have launched clinical trials using the patient's own stem cells to strengthen the heart which include umbilical cord blood and bone marrow stem cells. First, the umbilical cord blood clinical trial will be discussed [70].

Before the patient is even born, it is possible to diagnose the patient with HLHS. This is done in utero after week 20 of a pregnancy. This congenital heart disease requires a three-stage surgical repair including procedures called the Norwood, Glenn, and Fontan operation. However, this clinical study is assessing the idea of regenerative medicine after the stage 2 of the three- stage surgical repair. At the time of birth, the umbilical cord is cut, and blood is drained from it and processed in the laboratory as the infant is receiving the Norwood procedure, stage 1 . The cord blood is centrifuged, and the mononuclear cells are concentrated and frozen to be stored for future use. Then these cells are used in the second surgery and directly injected in the heart muscle. These cells release growth factors and act as a jump-start to regenerate the heart muscle [71]. Previously discussed is an overview of the umbilical cord study and its mechanisms, now further detail will be stated regarding this study.

The study reviewed above is one of the limited numbers of clinical trials that have assessed the efficacy of stem cell therapy in pediatric patients with congenital heart defects and have shown encouraging results in patients with HLHS. This is one of the two ongoing trials for regenerative medicine for patients with HLHS and they are both in phase 1. The umbilical cord study produced direct intramyocardial injection of umbilical-cord-blood-derived mononuclear cells in an infant with hypoplastic left heart syndrome undergoing stage-II surgical palliation [69]. Since the study is in phase 1 , this means it is being tested for its safety, side effects, and risks/benefits. Currently, the study is testing the safety and feasibility of injections of the autologous umbilical cord blood (UCB) cells into the patient. This is being completed at the time that the patient is undergoing surgery. As stated before, the stage-II surgical palliation, this is known as the Glenn surgical procedure [72]. The Glenn surgical operation occurs usually within six months of birth. During this procedure, the superior vena cava is disconnected from the heart and attached to the pulmonary artery. The purpose is to get deoxygenated blood from the upper body and take it to the lungs without passing through the heart [73].

The family was admitted to Mayo Clinic's phase-I study of autologous umbilical-cord blood cells for HLHS. The umbilical-cord blood was collected at the time of delivery which showed that these cells were easily accessible. Before the therapy, the infant received the Norwood procedure which is stage one of staged reconstruction. The Norwood procedure usually occurs within several days of birth depending on the heart defect. The goal of this operation is to make sure that blood-flow is controlled to prevent further damage to the heart and lung and will keep the patient alive until the second operation, the Glenn procedure [73]. Therefore, after the patient received this stage 1 procedure, the infant was discharged with no complications. As the infant developed, complications did start arising with reduced right ventricular systolic function and underwent cardiac catheterization as well as stage-II surgical palliation. During this procedure, an autologous cell-based, manufactured product was transplanted into the right ventricular free wall. The cells were delivered in $0.6 \mathrm{ml}$ of a solution containing 30 million cells/ml. No complicated occurred during these injections. After this operation, there was a persistent, stable, right ventricular systolic dysfunction shown and the infant was eventually discharged. At 1 and 3 months after autologous cell; delivery, the infant was evaluated.

Outpatient 24- hour did not demonstrate any arrhythmia. At 1 month, the results consisted of a NT-PRo-BNP level of $1175 \mathrm{pg} / \mathrm{mL}$ and a CK-MB level of $4.1 \mathrm{ng} / \mathrm{mL}$ and troponin T was undetectable. At 3 months, the transthoracic echocardiography showed improvement in the right ventricular systolic function with an estimated ejection fraction of $50 \%$ from $30-35 \%$ before the injection. This report gave an encouraging finding at the 3-month evaluation when right ventricular ejection fraction showed a great improvement shown in Figure 5 [69]. As stated before, the early phases consisting of phase 1 and 2 are done for researchers to test the safety of the procedure. Later, phase 3 will be conducted which studies if the treatment works better than the current standard therapy, surgery. By conducting this study using autologous umbilical cord blood, it will be determined if they can be used to strengthen the muscle of the right side of the patient's heart. This will result in using regenerative therapy for an additional treatment for the management of HLHS and other congenital heart defects [72].

\section{Clinical study using bone marrow stem cells}

The next study conducted by the Todd and Karen Wanek Family Program for Hypoplastic Left Heart Syndrome program used bone marrow stem cell in a clinical trial to determine if these stem cells from the patient's own bone marrow could be used the strengthen the heart. This investigation will help determine the safety and feasibility of regenerative therapy further for patients with this complex defect. Here, they used bone marrow from an individual's own body and processed it in the laboratory as well. The blood was separated and centrifuged just as was completed in the umbilical cord study. In the centrifuge, the various cell types are separated, making it easy to extract the stem cells, the mononuclear cells. These cells are processed and placed in a catheter into the femoral artery of the patient which go into the coronary blood vessels. Through the coronary blood supply,

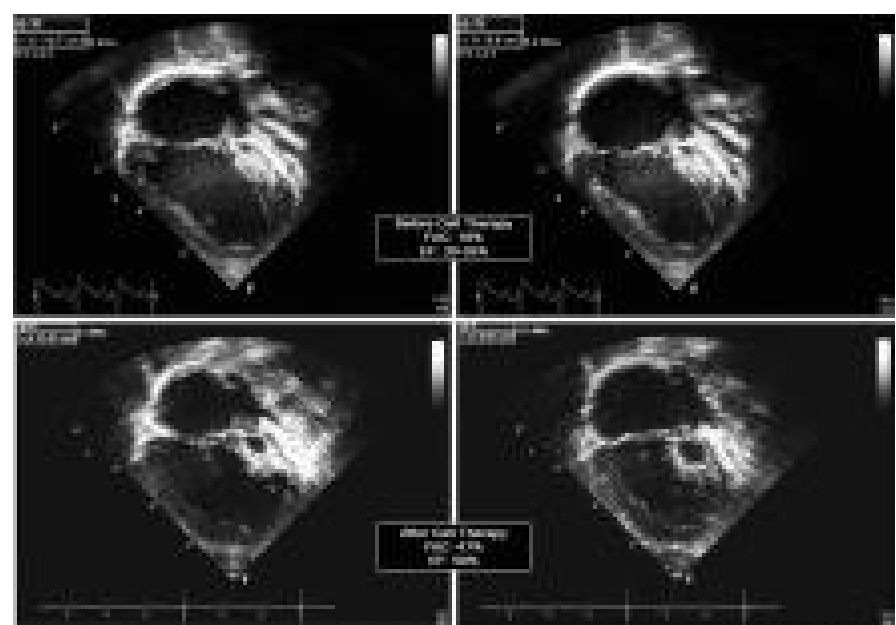

Figure 5. The results above show an echocardiogram before (top photos) and after (below photos) after 3 months of intramyocardial injection of umbilical-cord-blood-derived stem cells. This figure shows the improvement of the right ventricle functionality and are increased ejection fraction from $30-35 \%$ to $50 \% 69$ 
these stem cells are efficiently transferred to the heart entering into the blood vessels. As in the umbilical cord blood study, the stem cells also release growth factors that act as a jump start to stimulate new heart muscle cells to grow bigger. This clinical trial was performed in a young adult whereas the umbilical cord study is aimed to help young infants [71].

\section{Onoging and future clinical trials}

Many ongoing studies are being conducted to better understand what happens to the heart when the stem cells are injected directly into muscle in the right side of the heart. These are being performed in patients with HLHS during Stage II Glenn operation. A Phase IIb trial is being conducted with 100 participants of intramyocardial injects of UCBs that will begin in April 2019 and is estimated to end in February 2026 [74].

Another study is in the recruiting phase to assess the safety, tolerability, and efficacy of Longerveron Mesenchymal Stem Cells (LMSCs) during the stage II Glenn procedure. With a total of 30 patients, two stages with three cohorts will be performed. In the first stage, Cohort A of ten consecutive HLHS patients will be treated with LMSCs. The second stage will be double- blinded and twenty patients with HLHS will be randomized and treated with LMSCs. This study started on February 21, 2018 and plans to be completed in February 2022 [75].

There are also future clinical trials that are in place but are not recruiting thus far. One includes a trial that estimates to have 30 participants with hypoplastic left heart syndrome. During the patient's stage 2 procedure, the patient will be given previously harvested, isolated, and expanded autologous c-kit+ cardiac stem cells into the aorta. This study is planned to begin in July 1, 2019 and end in July 2025 [76].

\section{Considerations of stem cell therapy for CHD}

Congenital heart disease patients provide a different set of challenges as seen in patients with ischemic heart disease regarding stem cell therapy. Investigators are faced with designed studies that correlate with published literature. The underlying questions are what type of stem cell should be used in patients with CHD and the method of delivery of those stem cells. Many factors come into play when choosing the stem cells and delivery method such as the age of the patients, the diagnosis, and whether or not a surgical procedure is planned to occur at the time of administration of cells to provide direct exposure of cardiac structures. The indication for stem cell therapy in patients with CHD remains to be defined and many questions are needed to be answers to provide to greatest efficacy in the treatment of these challenging patients [18].

As stated before, there are many uncertainties related to stem cell therapies to treat patients with CHD and adult heart disease. Recent preclinical studies showed that angiogenetic factors secreted by MSCs may increase capillary density when a pulmonary vascular endothelial cell are transplanted in a model of pulmonary hypertension. However, recent advances in technology may enable us to understand the basis of myocardial adaptations to stem cell therapies in CHD. Children have similar symptoms as adults with ischemic cardiomyopathy that has been studied for stem cell therapy, however children with $\mathrm{CHD}$ may have unique structural changes that needs distinct reparative mechanisms [22].

\section{Conclusions}

In recent years, stem cell therapy has received recognition and begun to show encouraging results because of the idea that it will help long-term congenital heart disease and prevent the need for cardiac transplantation. It is seemed that stem cell therapy will provide benefits over surgery in terms of the quality of life and the heart's function. During the past decade, using stem cell therapy for the improvement of the cardiovascular system has improved tremendously. The future is likely to contain many improvements of the safety and effectiveness of stem cell therapy. Most studies focus patients with hypoplastic left heart syndrome and ongoing research is striving for a deeper understanding of the treatment more specifically. The reason for this is because HLHS is a complex form of CHD that requires a three-staged heart reconstruction and is usually left with heart transplantation being the last option. The hope is to use this emerging technology in stem cells to address the major issues in congenital heart disease that limit the lifespan and quality of life in so many children and adults. CHD represents the main medical heath care burden in the world with heart transplantations being the last option.

With the use of stem cell therapy, it could create a different option of treatment. Although surgical results for treatment of congenital heart disease and in specific, HLHS with the three- staged reconstruction, has improved and continued to advance, patients still are at significant risk for developing heart failure and thus needing cardiac transplantation. This therapy holds a great promise as an approach for CHD patients, but clinical research is necessary to understand how to apply it. The hope in using regenerative medicine is to minimize the risk of longterm ventricular failure. The experience with stem cell therapy in patients with congenital heart disease is limited. However, new insights into CPCs and MSCs isolated from pediatric patients may provide the foundation for exciting regenerative approaches for CHD treatments. There is a long way to come to have stem cell therapy become a fully established treatment since more research and clinical trials are necessary. There are ongoing and proposed clinical trials that are being conducted which will help certify the efficacy of this therapeutic treatment.

Since the heart has a limited availability to self-renew, CHD is the most prevalent congenital anomaly. This review summarized recent advances in the field of cardiac cell therapies. Deeper understanding of stem cell therapy use in damaged heart could lead to the development of regenerative medicines for the pediatric patients with this disease.

\section{References}

1. Biehl JK, Russell B (2009) Introduction to stem cell therapy. J Cardiovasc Nurs 24: 98-103. [Crossref]

2. Van der Linde D, Konings EE, Slager MA (2011) Birth prevalence of congenital heart disease worldwide: A systematic review and meta-analysis. J Am Coll Cardiol. 58: 2241-2247.

3. Pleva L, Kukla P, Vítková K, Procházka V (2019) Rationale and design of a prospective, randomised study of retrograde application of bone marrow aspirate concentrate (BMAC) through coronary sinus in patients with congestive heart failure of ischemic etiology (the RETRO study). BMC Cardiovasc Disord 19: 1011-1019.

4. Tsilimigras DI, Oikonomou EK, Moris D, Schizas D, Economopoulos KP, et al. (2017) Stem cell therapy for congenital heart disease: A systematic review. Circulation 136: 2373- 2385.

5. Sheng CC, Zhou L, Hao J (2013) Current stem cell delivery methods for myocardial repair. Biomed Res Int 2013: 547902. [Crossref]

6. Collins JM, Russell B (2009) Stem cell therapy for cardiac repair. J Cardiovasc Nurs 24: 93-97. [Crossref] 
7. Ambastha C, Bittle GJ, Morales D (2018) Regenerative medicine therapy for single ventricle congenital heart disease. Transl Pediatr 7: 176-187.

8. Bosman A, Edel MJ (2015) Bioengineering and stem cell technology in the treatment of congenital heart disease. J Clin Med 4: 768-781. [Crossref]

9. Si M, Ohye RG (2017) Stem cell therapy for the systemic right ventricle. Expert Review of Cardiovascular Therapy 15: 813-823.

10. Avolio E, Caputo M, Madeddu P (2015) Stem cell therapy and tissue engineering for correction of congenital heart disease. Front Cell Dev Biol 3: 1-17.

11. Roberts EG, Piekarski BL, Huang K, Emani S (2018) Evaluation of placental mesenchymal stem cell sheets for myocardial repair and regeneration. Tissue Eng Part A 2: 1-10. [Crossref]

12. Mantakaki A, Fakoya AOJ, Sharifpanah F (2018) Recent advances and challenges on application of tissue engineering for treatment of congenital heart disease. Peer $J 6$ : e5805

13. Wehman B, Kaushal S (2015) The emergence of stem cell therapy for patients with congenital heart disease. Circulation Research 116: 566-569.

14. Oh H, Ito H, Sano S (2016) Challenges to success in heart failure: Cardiac cell therapies in patients with heart diseases. $J$ Cardiol 68: 361-367. [Crossref]

15. Ghafarzadeh M, Namdari M, Eatemadi A (2016) Stem cell therapies for congenital heart disease. Biomed Pharmacother 84: 1163-1171. [Crossref]

16. Galdos FX, Guo Y, Paige SL, VanDusen NJ, Wu SM, et al. (2017) Cardiac regeneration: lessons from development. Circ Res 120: 941-959. [Crossref]

17. Duelen R, Sampaolesi M (2017) Stem Cell Technology in Cardiac Regeneration: A Pluripotent Stem Cell Promise. EBioMedicine 16: 30-40. [Crossref]

18. Wehman B, Kaushal S (2015) The emergence of stem cell therapy for patients with congenital heart disease. Circulation Research 116: 566-569.

19. Files MD, Boucek RJ (2012) 'Shovel-Ready' applications of stem cell advances for pediatric heart disease. Curr Opin Pediatr 24: 577-583. [Crossref]

20. Bernstein HS, Srivastava D (2012) Stem cell therapy for cardiac disease. Pediatr Res 71: 491-499. [Crossref]

21. Kimura N (2018) Regenerative therapy for patients with congenital heart disease. Keio J Med 2: 1-10. [Crossref]

22. Oh H (2017) Cell therapy trials in congenital heart disease. Circulation Research 120 1353-1366.

23. Chan HC, Chiu WT, Wu JM, Wang JN, Wong TK (2014) P345Isolation and identification of a new source of human stem cells expanded from pediatric congenital heart disease. Cardiovascre 103: S63.

24. Li C, Matsushita S, Li Z, Guan J, Amano A (2017) C-kit positive cardiac outgrowth cells demonstrate better ability for cardiac recovery against ischemic myopathy. J Stem Cell Res Ther 7: 7633.1000402.

25. Singla DK (2016) Stem cells and exosomes in cardiac repair. Curr Opin Pharmaco 27: 19-23. [Crossref]

26. Peral S, Burkhart H, Nelson T (2014) Utilization of stem cells to treat congenital heart disease. Current Opinion in Pediatrics 26: 553-560.

27. Vanover M, Wang A, Farmer D (2017) Potential clinical applications of placental stem cells for use in fetal therapy of birth defects. Placenta 59: 107-112.

28. Bittle GJ, Wehman B, Karathanasis SK, Kaushal S (2017) Clinical progress in cell therapy for single ventricle congenital heart disease. Circ Res 120: 1060-1062.

29. Wehman B, Siddiqui OT, Mishra R, Sharma S, Kaushal S (2015) Stem cell therapy for CHD: towards translation. Cardiol Young 25: 58-66. [Crossref]

30. Ghafarzadeh M, Namdari P, Tarhani M, Tarhani F (2018) A review of application of stem cell therapy in the management of congenital heart disease. J Matern Fetal Neonatal Med 2: 1-10. [Crossref]

31. Pincott ES, Burch M (2012) Potential for stem cell use in congenital heart disease. Future Cardiol 8: 161-169. [Crossref]

32. Amini H, Rezaie J, Vosoughi A, Rahbarghazi R, Nouri M (2017) Cardiac progenitor cells application in cardiovascular disease. J Cardiovasc Thorac Res 9: 127-132.

33. Ghazizadeh Z, Vahdat S, Fattahi F (2015) Isolation and characterization of cardiogenic, stem- like cardiac precursors from heart samples of patients with congenital heart disease. Life Sci 137: 105-115.
34. Claudia Bearzi, Marcello Rota, Toru Hosoda (2007) Human cardiac stem cells. Proc Natl Acad Sci USA 104: 14068-14073. [Crossref]

35. Wehman B, Sharma S, Mishra R, Guo Y, Colletti EJ, et al. (2015) Pediatric end-stage failing hearts demonstrate increased cardiac stem cells. Annals of Thoracic Surgery 100: 615-622.

36. Hinton RB, Ware SM (2017) Heart failure in pediatric patients with congenital heart disease. Circ Res 120: 978-994. [Crossref]

37. Tarui S, Ishigami S, Ousaka D (2015) Transcoronary infusion of cardiac progenitor cells in hypoplastic left heart syndrome: Three-year follow-up of the transcoronary infusion of cardiac progenitor cells in patients with single-ventricle physiology (TICAP) trial. $J$ Thorac Cardiovasc Surg 150: 2

38. Ishigami S, Ohtsuki S, Eitoku T (2017) Intracoronary cardiac progenitor cells in single ventricle physiology: The PERSEUS (cardiac progenitor cell infusion to treat univentricular heart disease) randomized phase 2 trial. Circ Res 120: 1162-1173.

39. Bulatovic I, Månsson-Broberg A, Sylvén C, Grinnemo K (2016) Human fetal cardiac progenitors: The role of stem cells and progenitors in the fetal and adult heart. Best Practice \& Research Clinical Obstetrics \& Gynaecology 31: 58-68.

40. Types of stem cells ? A closer look at stem cells. [Available] https://www. closerlookatstemcells.org/learn-about-stem-cells/types-of-stem-cells/.

41. Lo B, Parham L (2009) Ethical issues in stem cell research. Endocr Rev 30: 204-213. [Crossref]

42. Lambert V, Gouadon E, Capderou A (2015) Right ventricular failure secondary to chronic overload in congenital heart diseases: Benefits of cell therapy using human embryonic stem cell- derived cardiac progenitors. J Thorac Cardiovasc Surg 149: 715. e1.

43. Velasquez-Mao A, Tsao CJM, Monroe MN (2017) Differentiation of spontaneously contracting cardiomyocytes from non-virally reprogrammed human amniotic fluid stem cells. PLOS ONE 12: e0177824.

44. Weber B, Kehl D, Hoerstrup SP (2014) Amniotic fluid-derived cells: An autologous cell source for cardiovascular tissue engineering. Perinatal Stem Cells 9: 99-105.

45. Kunisaki SM (2012) Congenital anomalies: Treatment options based on amniotic fluidderived stem cells. Organogenesis 8: 89-95.

46. Kunisaki SM (2018) Amniotic fluid stem cells for the treatment of surgical disorders in the fetus and neonate. Stem Cells Transl Med 7: 767-773. [Crossref]

47. Induced pluripotent stem cells (iPS) [Available] https://stemcell.ucla.edu/inducedpluripotent-stem-cells.

48. Schmidt D, Achermann J, Odermatt B (2007) Prenatally fabricated autologous human living heart valves based on amniotic fluid derived progenitor cells as single cell source. Circulation 116: 64.

49. Bittle GJ, Morales D, Deatrick KB (2018) Stem cell therapy for hypoplastic left heart syndrome: Mechanism, clinical application, and future directions. Circ Res 123: 288300.

50. Weiss DJ, Casaburi R, Flannery R, LeRoux-Williams M, Tashkin DP (2013) A placebocontrolled, randomized trial of mesenchymal stem cells in COPD. Chest 143: 15901598 .

51. Zhong J, Wang S, Shen WB, Kaushal S, Yang P (2018) The current status and future of cardiac stem/progenitor cell therapy for congenital heart defects from diabetic pregnancy. Pediatr Res 83: 275-282.

52. Gao Y, Jacot JG (2015) Stem cells and progenitor cells for tissue-engineered solutions to congenital heart defects. Biomark Insights 10: 139-146.

53. Amado LC, Saliaris AP, Schuleri KH (2005) Cardiac repair with intramyocardial injection of allogeneic mesenchymal stem cells after myocardial infarction. Proc Natl Acad Sci USA 102: 11474-11479.

54. Wang S, Huang S, Gong L (2018) Human neonatal thymus mesenchymal stem cells promote neovascularization and cardiac regeneration. Stem Cells Int $8: 1-7$.

55. Hatzistergos KE, Saur D, Seidler B, Balkan W, Breton M, et al. (2016) Stimulatory effects of mesenchymal stem cells on cKit + cardiac stem cells are mediated by SDF1/ CXCR4 and SCF/cKit signaling pathways. Circ Res 119: 921-930. [Crossref]

56. Rupp S, Bauer J, Tonn T (2009) Intracoronary administration of autologous bone marrow- derived progenitor cells in a critically ill two-yr-old child with dilated cardiomyopathy. Pediatr Transplant 13: 620-623.

57. Tarui S, Sano S, Oh H2 (2014) Stem cell therapies in patients with single ventricle physiology. Methodist Debakey Cardiovasc J 10: 77-81. [Crossref] 
58. Nelson TJ, Cantero Peral S (2016) Stem cell therapy and congenital heart disease. $J$ Cardiovasc Dev Dis 3. [Crossref]

59. Bartolucci J, Verdugo FJ, Gonzalez PL (2017) Safety and efficacy of the intravenous infusion of umbilical cord mesenchymal stem cells in patients with heart failure: A phase 1/2 randomized controlled trial (RIMECARD trial [randomized clinical trial of intravenous infusion umbilical cord mesenchymal stem cells on cardiopathy]). Circ Res 121: 1192-1204.

60. Marbán E (2014) Breakthroughs in cell therapy for heart disease: focus on cardiospherederived cells. Mayo Clin Proc 89: 850-858. [Crossref]

61. Miyagawa S, Sawa Y (2018) Building a new strategy for treating heart failure using Induced Pluripotent Stem Cells. J Cardiol 72: 445-448. [Crossref]

62. Kastenberg ZJ, Odorico JS (2008) Alternative sources of pluripotency: science, ethics, and stem cells. Transplant Rev (Orlando) 22: 215-222. [Crossref]

63. Yang C, Al-Aama J, Stojkovic M (2015) Concise review: Cardiac disease modeling using induced pluripotent stem cells. Stem Cells 33: 2643-2651.

64. Doyle MJ, Lohr JL, Chapman CS, Koyano-Nakagawa N, Garry MG, et al. (2015) Human induced pluripotent stem cell-derived cardiomyocytes as a model for heart development and congenital heart disease. Stem Cell Rev 11: 710-727.

65. Jiang W, Zhang, Feng Lan, Hongjia (2016) Human induced pluripotent stem cells for inherited cardiovascular diseases modeling. Current Stem Cell Research \& Therapy 11: $1-10$.

66. Maher KO, Xu C (2013) Marching towards regenerative cardiac therapy with human pluripotent stem cells. Discov Med 15: 349-356.

67. Liu B, Lee BW, Nakanishi K (2018) Cardiac recovery via extended cell-free delivery of extracellular vesicles secreted by cardiomyocytes derived from induced pluripotent stem cells. Nat Biomed Eng 2: 293-303.
68. Yerebakan C, Sandica E, Prietz S (2009) Autologous umbilical cord blood mononuclear cell transplantation preserves right ventricular function in a novel model of chronic right ventricular volume overload. Cell Transplant 18: 855-868.

69. Burkhart HM, Qureshi MY, Peral SC (2015) Regenerative therapy for hypoplastic left heart syndrome: First report of intraoperative intramyocardial injection of autologous umbilical-cord blood-derived cells. J Thorac Cardiovasc Surg 149: 35.

70. Center for regenerative medicine - hypoplastic left heart syndrome. [Available] http:// www.mayo.edu/research/centers-programs/center-regenerative-medicine/focus-areas/ hypoplastic-left-heart-syndrome.

71. Todd and karen wanek family program for hypoplastic left heart syndrome (hlhs) clinical trials. [Available] http://www.mayo.edu/research/centers-programs/toddkaren-wanek-family-program-hypoplastic-left-heart-syndrome/hlhs-research-patientcare/clinical-trials.

72. A safety study of autologous umbilical cord blood cells for treatment of hypoplastic left heart syndrome. [Available] http://www.mayo.edu/research/clinical-trials/cls20450452

73. The children's hospital of. staged reconstruction heart surgery. [Available] https:// www.chop.edu/treatments/staged-reconstruction-heart-surgery.

74. Nelson T (2019) Intramyocardial injection of autologous umbilical cord blood derived mononuclear cells during surgical repair of hypoplastic left heart syndrome (AutoCell-S2). https://clinicaltrials.gov/ct2/show/NCT03779711.

75. Longeveron L (2019) Longeveron mesenchymal stem cells (LMSCs) delivered during stage II surgery for hypoplastic left heart syndrome (ELPIS) [Available] https:// clinicaltrials.gov/ct2/show/NCT03525418.

76. Hare J (2019) Autologous cardiac stem cell injection in patients with hypoplastic left heart syndrome: An open label pilot study [Available] https://clinicaltrials.gov/ct2/ show/NCT03406884.

Copyright: $(02019$ Chase DM. This is an open-access article distributed under the terms of the Creative Commons Attribution License, which permits unrestricted use, distribution, and reproduction in any medium, provided the original author and source are credited. 